Original Article

\title{
Tailored patient self-management and supervised, home-based, pulmonary rehabilitation for mild and moderate chronic obstructive pulmonary disease
}

\author{
Teresa Paolucci ${ }^{1)^{*}}$, Letizia Pezzi ${ }^{2)}$, Rosa Grazia Bellomo ${ }^{3)}$, Antonella Spacone ${ }^{4)}$, \\ Niki Giannandrea ${ }^{4)}$, Andrea Di Matteo ${ }^{1)}$, Pierpaolo Prosperi ${ }^{4)}$, Andrea Bernetti ${ }^{5)}$, \\ Massimiliano Mangone ${ }^{5)}$, Francesco Agostini ${ }^{5)}$, Raoul Saggini ${ }^{1}$ ) \\ 1) Unit of Physical Medicine and Rehabilitation, Department of Oral Medical Science and \\ Biotechnology (DSMOB), Gabriele d'Annunzio University of Chieti-Pescara: Viale Abruzzo 322, \\ $66100 \mathrm{CH}$, Chieti-Pescara, Italy \\ 2) U.O. Specialistic Rehabilitation, Hospital Cremona, Italy \\ 3) Department of Biomolecular Sciences, University of Study of Urbino Carlo Bo, Italy \\ 4) U.O.C. Pneumology and Respiratory Physiopathology, Hospital "S. Spirito" Pescara, Italy \\ 5) Physical Medicine and Rehabilitation Unit, Department of Anatomical and Histological Sciences, \\ Legal Medicine and Orthopedics, Sapienza University of Rome, Italy
}

\begin{abstract}
Purpose] Our study aimed to estimate the effects of a supervised, intensive, home-based-pulmonaryrehabilitation (HBPR) program for mild and moderate chronic-obstructive-pulmonary-disease (COPD) patients. [Participants and Methods] A retrospective, case-control study. Forty-three $(\mathrm{N}=43)$ patients were observed, divided into Treatment-Group (TG) and Control-Group (CG). The TG (N=23) underwent a 4-week training program, consisting of endurance and strength training sessions, performed 4-times per week. In addition, inspiration muscle training was performed, with a threshold device. The primary outcome was dyspnea improvements, measured by the modified-Borg-scale (mBS), and the secondary outcome was the determination of diaphragm excursion and function, using ultrasound (US) assessment to measure clinical parameters. [Results] The results suggested significantly improved mBS scores, measured for the $\Delta \mathrm{T} 0-\mathrm{T} 1$ and $\Delta \mathrm{T} 0-\mathrm{T} 2$ time points; improved diaphragm-excursion (Dia-Ex) at $\Delta \mathrm{T} 0-\mathrm{T} 1$ and $\Delta \mathrm{T} 0-\mathrm{T} 2$; and improved maximum Dia-Ex at $\Delta \mathrm{T} 0-\mathrm{T} 1$ and $\Delta \mathrm{T} 1-\mathrm{T} 2$ in the $\mathrm{TG}$ compared with the CG. Moreover, the results showed improvements over time for all parameters in the TG versus CG, suggesting a constant improvement in respiratory pathology. [Conclusion] A supervised HBPR plan was effective in reducing dyspnea by the $\mathrm{mBS}$, and improving diaphragmatic function, as determined by US evaluation, and lastly improving quality of life in patients with mild-to-moderate COPD. Key words: Rehabilitation, Quality of life, Ultrasound
\end{abstract}

(This article was submitted Jul. 13, 2021, and was accepted Oct. 25, 2021)

\section{INTRODUCTION}

Chronic obstructive pulmonary disease (COPD) has a high and growing prevalence, globally, and is a leading cause of mortality and morbidity ${ }^{1}$. Unfortunately, COPD is often undiagnosed during the initial phase; therefore, the prevalence is likely underestimated, resulting in delayed access to treatment and care pathways, especially rehabilitative options. During

*Corresponding author. Teresa Paolucci (E-mail: teresapaolucci@hotmail.com)

(C2022 The Society of Physical Therapy Science. Published by IPEC Inc.

(c) (1) $\odot$ This is an open-access article distributed under the terms of the Creative Commons Attribution Non-Commercial No Deriva(CY NG ND tives (by-nc-nd) License. (CC-BY-NC-ND 4.0: https://creativecommons.org/licenses/by-nc-nd/4.0/) 
the mild or early stages of the disease, the symptoms often do not interfere with daily living activities ${ }^{2-4)}$.

COPD is a lung disease, characterized by airflow limitations, and is not currently fully reversible ${ }^{2}$. When the patients progress beyond the initial stages and develop the more severe stages of COPD, the activation of accessory expiratory muscles becomes an increasingly apparent functional compensation, associated with increased energy expenditure and muscle fatigue ${ }^{5}$. During progression, from mild to severe stages of COPD, a gradual reduction in diaphragm muscle activation can be detected. Muscle weakness and respiratory fatigue appear to be associated with muscle wasting and a shift in the muscle fiber composition, from slow to fast, respectively ${ }^{6}$. Despite the engagement of accessory expiratory muscles, reduced lung elasticity and mucus stagnation can contribute to an associated emphysematous situation in many patients ${ }^{5)}$. During the last decade, drug therapy options for COPD patients have improved dramatically; however, treatment that is integrated with rehabilitation exercises has been associated with longer-lasting results. Respiratory rehabilitation involves exercise training, education, nutritional therapy, psychological support, and the promotion of self-management techniques such as breathing and relaxation technique (i.e., yoga pranayama respiration, diaphragmatic breathing training, tai chi, and qui gong ${ }^{7-9)}$.

Home-based, pulmonary rehabilitation (HBPR) therapy has been demonstrated to be a clinically and cost-effective approach to minimizing COPD symptoms. Traditional, in-clinic pulmonary rehabilitation (PR) programs are not sufficient to treat the entire COPD population, as patients may encounter transportation difficulties or have other physical limitations that prevent their regular participation in traditional rehabilitation programs ${ }^{10,11)}$.

HBPRs have emerged as valuable strategies for improving the clinical outcomes of COPD, such as dyspnea, exercise capacity, and health-related quality of life ${ }^{12)}$. COPD patients, through participation in HBPRs, could access education and practice and improve self-management techniques within the familiar and known settings in which they perform their normal activities of daily life, a very important topic in chronic diseases ${ }^{10)}$. Some authors have suggested that HBPRs can have beneficial effects for patients with COPD, not only in terms of increased exercise tolerance and improved quality of life but also by contributing to the regulation of systemic inflammation, increased inspiratory muscle strength, and decreased serum interleukin (IL)-8 levels ${ }^{13)}$. The rehabilitative management of COPD patients and respiratory kinesitherapy are designed to improve the quality of life of patients in the short and medium term and reduce the progression of chronic COPD pathology and limit exacerbations over the long term, as far as possible. In this sense, rehabilitation takes on a double meaning: treatment and support to drug therapy but also intense prevention as a slowdown in the progression of the clinical situation, albeit in its chronicity ${ }^{13)}$.

Also, Maltais et al. suggested that although supervised outpatient pulmonary rehabilitation (PR) remains the standard form of rehabilitative care, it is a less effective alternative than home-based alternatives, especially for those patients who are unable to attend PR regularly ${ }^{12}$. For HBPR to be effective, according to other authors, it should be well structured and organized, with regular contact, at least by phone, between the physiotherapist and the COPD patients. COPD patients tend to value the convenience of HBPRs and have reported experiencing positive impacts, in terms of improved physical fitness and reduced symptoms, and felt better supported than during center based $\mathrm{PR}^{14)}$. Often, HBPRs utilize non-intensive protocols, based on 2 to 3 sessions per week, for approximately 2 months. In addition, although the PR programs are standardized, they often do not include specific inspiratory muscle training (IMT) or devices when implemented at home ${ }^{7,14,15)}$.

Gosselink and colleagues suggested that IMT could represent an effective rehabilitative treatment modality in COPD patients ${ }^{15}$. Recently, Smargiassi et al. demonstrated the potential role of ultrasound (US) measurements to determine diaphragm thickness at the end of a maximal inspiration ${ }^{14}$. Moreover, the US-based analysis of diaphragmatic excursion has been correlated with bronchial obstruction and dyspnea. Crimi et al. performed a prospective, observational study to assess the routine use of US diaphragmatic assessment before and after PR and recommended its use as an additional tool for the evaluation of the clinical effects of PR in COPD patients ${ }^{16,17)}$.

Diaphragm US measurements have also been widely used in the clinical setting to assess diaphragm movement amplitudes and thickness in humans who are affected by various neuromuscular conditions ${ }^{18-20)}$. Diaphragm weakness is indicated by a lower-than-normal amplitude of excursion during deep breathing, with or without paradoxical movement on sniffing ${ }^{21,22)}$. In patients with COPD, neuromechanical dissociation occurs, due to dynamic hyperinflation, which causes the breathing pattern to become more rapid and shallow 22,23$)$.

We hypothesized that an intensive and supervised HBPR could improve COPD dyspnea and quality of life when performed at home, with constant, weekly support from a physiotherapist. As suggested by Crimi et al., we evaluated the diaphragmatic excursion by US to monitor the biomechanical effects of HBPR on the diaphragm muscle ${ }^{16)}$. Therefore, our study aimed to estimate the effects of an intensive and supervised HBPR in mild and moderate COPD patients. The principal outcome was dyspnea improvement, as assessed by the modified Borg scale (mBS), whereas the secondary outcome was diaphragm function and excursion, as assessed by US-based measurements of clinical parameters.

\section{PARTICIPANTS AND METHODS}

A retrospective, case-control study was conducted per the STROBE guideline to determine the effects of specific, intensive, and supervised HBPR exercises in COPD patients on dyspnea and diaphragmatic excursion, evaluated by the $\mathrm{mBS}$ and US-based measurements, respectively ${ }^{24)}$.

This study was performed from February 2019 to November 2019, at the U.O.C. Pneumology and Respiratory Physio- 
pathology of Hospital "S. Spirito" Pescara (Italy), in collaboration with the Rehabilitation Unit of IRCCS "Bonino Pulejo", Messina, (Italy).

The Ethics Committee of IRCSS - Bonino-Pulejo approved the protocol, and the study was approved by the Local Department Committee of G. D’Annunzio University of Chieti ( $\left.\mathrm{N}^{\circ} 3350\right)$. This study conformed to Good Clinical Practice and all procedures followed the ethical standards of the Helsinki Declaration on human experimentation. All patients gave written informed consent, after receiving detailed information regarding the study's aims and procedures.

Observed patients were included consecutively and divided into 2 groups: The Control Group (CG) as usual care and home rehabilitation group constituted the Treatment Group (TG).

Patients aged over 55 years and diagnosed with mild-to-moderate COPD, were included in this study, according to the Global Initiative for Chronic Obstructive Lung Disease (GOLD) criteria ${ }^{25}$. Patients were also required to be clinically stable and free from disease exacerbation during the last 30 days.

The exclusion criteria were: severe comorbidities, such as heart diseases, orthopedic diseases in the upper and lower limbs, motor sequelae from neurological or visual disorders that interfered with the ability to perform physical exercises, uncontrolled hypertension, disease exacerbation during the program, smokers; and those who did not agree to participate in the study.

The pulmonologist identified all patients who met the inclusion/exclusion criteria and obtained signed informed consent forms for participation in this observational study. Subsequently, a physiatrist evaluation was performed to define the homebased rehabilitative plan. Evaluations were performed for each group before treatment (T0, baseline), at the end of the rehabilitation treatment (T1), and after 2 months of follow-up (T2).

The determination of diaphragm excursion and the measurement of diaphragm thickening were performed at all three evaluation points. To reduce variability and improve measurement reproducibility, the acquisition procedure was standardized. The US probe was positioned on the middle clavicle line, and a subcostal scan was performed to acquire the diaphragmatic excursion. Because the best US acoustic window is obtained near the liver parenchyma, scans were only performed for the right hemidiaphragm. To study the diaphragmatic thickness, the probe was positioned between the VIII and X intercostal space, between the anterior and middle axillary line.

The US evaluation was performed using two probes that are commonly used in US practice: a 3-6 MHz convex probe and a high-frequency linear probe, from 7-13 MHz, with a low-frequency, curvilinear, or phased-array transducer, using 2-dimensional brightness or the B-mode US, and M-mode US ${ }^{26-29)}$. An anatomical evaluation of thickness, measured using the linear probe, was performed during various phases of the respiratory cycle: i) spontaneous breathing; ii) maximal inspiration; iii) and maximal exhalation. The values were reported in $\mathrm{mm}$ for thickening and $\mathrm{cm}$ for excursion. Diaphragmatic excursion was evaluated using the convex probe.

For both measurements, the patient was placed in the supine position, with the back of the bed tilted at approximately $15^{\circ}$. Patients were asked to place their hands behind the nape of the neck, to facilitate the opening of the intercostal space and allow for the performance of intercostal scans.

A total of 5 measurements were acquired for each parameter, which were analyzed by discarding the two most extreme values and calculating an average based on the 3 remaining values. The measurement procedures were performed by a physiatrist with experienced performing muscle and respiratory US. The following reference values were used: diaphragm excursion during normal breathing, $1.8 \pm 0.3 \mathrm{~cm}$ for males and $1.6 \pm 0.3 \mathrm{~cm}$ for females; diaphragm excursion during maximal inspiration, $7.0 \pm 0.6 \mathrm{~cm}$ for males and $5.7 \pm 1.0$ for females; diaphragm thickening during normal breathing, $1.9 \pm 0.2 \mathrm{~mm}$; and diaphragm thickening during maximal inspiration, $4.9 \pm 0.5 \mathrm{~mm}^{28)}$.

Spirometry was performed to assess the forced vital capacity (FVC) and one-second forced expiratory volume $\left(\mathrm{FEV}_{1}\right)$, to stratify the degree of pulmonary dysfunction as mild, moderate, severe, or very severe $\left(\mathrm{FEV}_{1}>80 \%\right.$ was categorized as mild, between $50 \%$ to $80 \%$ as moderate, between $30 \%$ to $50 \%$ as severe, and $<30 \%$ as very severe $)^{30,31)}$. Maximal inspiratory pressure (MIP) and maximal expiratory pressure (MEP) were measured, as reliable parameters for the assessment of respiratory muscle strength ${ }^{32)}$.

The modified Borg scale (mBS) was used to assess dyspnea. The mBS is a graduated, non-linear scale, ranging from 0 to $10(0=\text { absence of dyspnea; } 10=\text { maximum dyspnea })^{33,34)}$.

To measure the speed and tolerance of walking, the 6-minute walking test (6-MWT) was used. This test measures the distance walked with maximal intensity, which reflects the patient's functional exercise abilities for daily physical activities better than conventional lung functional outcomes ${ }^{35-37)}$.

The COPD Assessment Test (CAT) was used to evaluate how COPD affects patients' lives ${ }^{38}$. The CAT measures health impacts on a scale from 0 to 40 (0-10 low, 11-20 medium, 21-30 high, and 31-40 very high). CAT $\geq 10$ corresponds to GOLD Groups B or D, whereas CAT $<10$ corresponds to GOLD Groups A or C.

The physiotherapist and physiatrist instructed the patient how to perform the exercise program at home, during the physiatrist examination. The exercise intensity was calculated for each individual, based on the target heart rate (HR), which was calculated using the Karvonen formula [(maximal HR - resting HR) $\times$ training $\%+$ resting HR], and the number of repetitions of each exercise to be performed was determined by the repeated administration of the fatigue resistance test (FRT), with one-minute rest intervals ${ }^{39}$ ). The FRT aimed to result in the failure of each activity, due to fatigue, from 40 to 60 sec after the beginning of each movement (extension and flexion of the knee, abduction and flexion of the shoulder, and the bending of 
the elbow). The physiotherapist asked the patient to perform the maximum number of repetitions of each movement, at the maximum range of motion (ROM) without pain, for up to 40-60 sec. The therapist recorded the number of repetitions and the time until fatigue.

After the first month, each patient also received a booklet describing the exercises to perform, which they were asked to perform twice per week, for at least $60 \mathrm{~min}$. The booklet also included an explanation regarding the disease and the importance making changes to motor activity lifestyle, nutrition, and physical activity ${ }^{40)}$.

Once per week, on a day of the patient's choosing, the physiotherapist visited the patient's home, to supervise the quality and performance of PR exercises and to suggest personal adaptations to the intensity of the exercise for each patient, as necessary, based on the physiotherapist's perception of the patient's dyspnea, in addition to the 4 weekly sessions of the rehabilitation program.

During the first month, the 4-week training program consisted of i) endurance and ii) strength training sessions, which lasted from 45 to 60 min each and were performed 4 times per week. Each of the 16 sessions consisted of a 10-min warm-up period, 20-40 min of aerobic activity, and a 10-min cool-down period.

The main objective of endurance training was to improve aerobic exercise capacity, via interval training (IT), which consists of a sequence of on-and-off, high-intensity, muscular loading exercises for the peripheral muscles, without overloading the cardiorespiratory system ${ }^{41)}$. The implementation of IT has been shown to facilitate high-intensity lower-limb exercises, which would not otherwise be tolerable.

The objective of strength training was to improve muscle mass making aerobic training easier to tolerate. The targeted muscle groups were the major muscles of locomotion (quadriceps, gluteal muscles, gastrocnemius), the major muscles of arm function (biceps, triceps, deltoids, trapezius, latissimus dorsi), and the abdominal muscles. Strength exercises were performed using elastic fitness bands, as illustrated in Figs. 1 and 2.

Between each series of strength exercises, the patient was allowed 2 minutes of rest ${ }^{39)}$. Each week, the training load increased by 1 series, for a total of 5 series at the end of the 4-week protocol (Table 1).

In addition, IMT was performed, which consisted of a treatment to improve respiratory muscle strength and endurance, which has been shown to reduce dyspnea when added to general exercise training ${ }^{15}$. Studies have shown the increased effectiveness of IMT strength training among patients with COPD, especially when combined with the use of a pressure threshold device $^{42}$ ), aiming to increase strength (assessed with the MIP), improve exercise tolerance (assessed with the 6-MWT), and reduce dyspnea (assessed with the $\mathrm{mBS}$ ). This pressure threshold device is characterized by a spring-independent, one-way valve, independent of the airflow, which ensures a fixed resistance $\left(\mathrm{cmH}_{2} \mathrm{O}\right)$. The resistance has a scale, ranging from 7 to $41 \mathrm{~cm} \mathrm{H} \mathrm{H}_{2} \mathrm{O}$, measured in increments of $2 \mathrm{~cm} \mathrm{H}_{2} \mathrm{O}$. The tension of the grooves causes the valve to open at a predetermined pressure. The device is designed to block flow below the threshold pressure, whereas the valve opens once pressure has been exceeded. At the end of the IMT sessions, stretching exercises and relaxation techniques were performed (Table 1).

During the daily home rehabilitative sessions, the patient was instructed by the doctor and physiotherapist to monitor

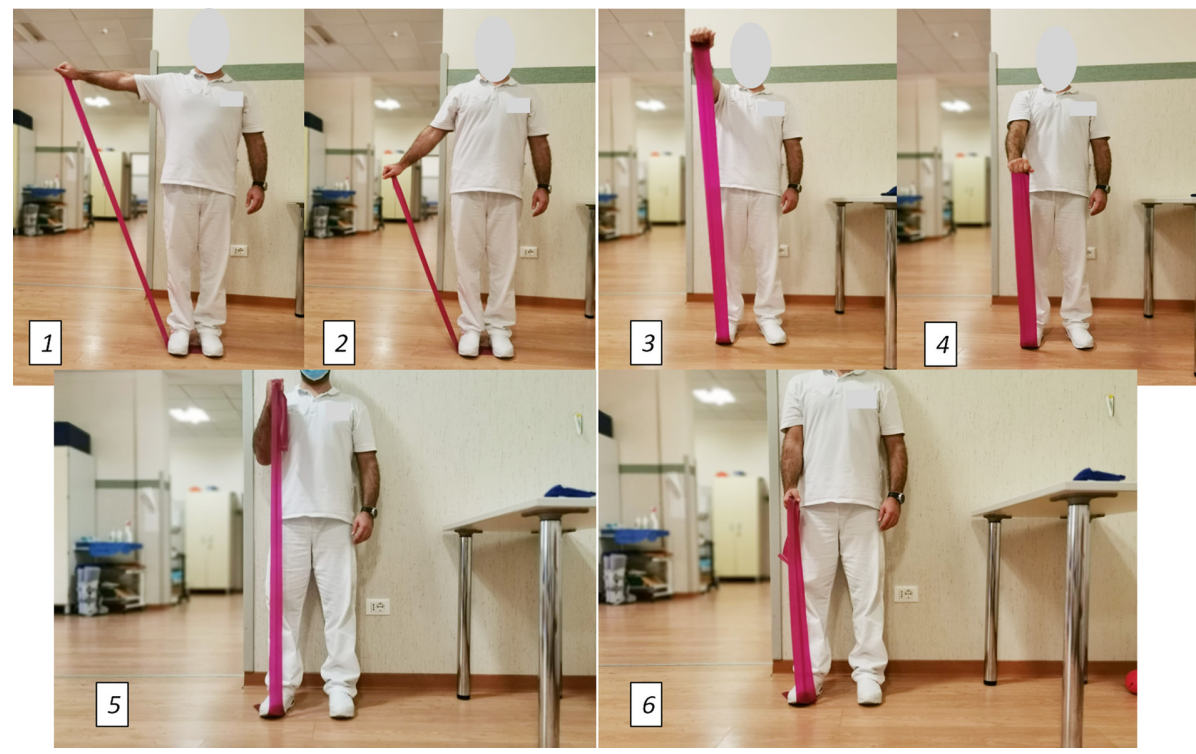

Fig. 1. Home-based exercises for upper limbs.

Exercises to strengthen the upper arm muscles (biceps, triceps, deltoids, trapezius, latissimus dorsi); 1-2: abduction-adduction exercises of the shoulder; 3-4: flexion exercises of the shoulder; 5-6: flexion exercises of the elbow (All the exercises illustrated must be performed with shoes with a rubber sole for patient safety). 


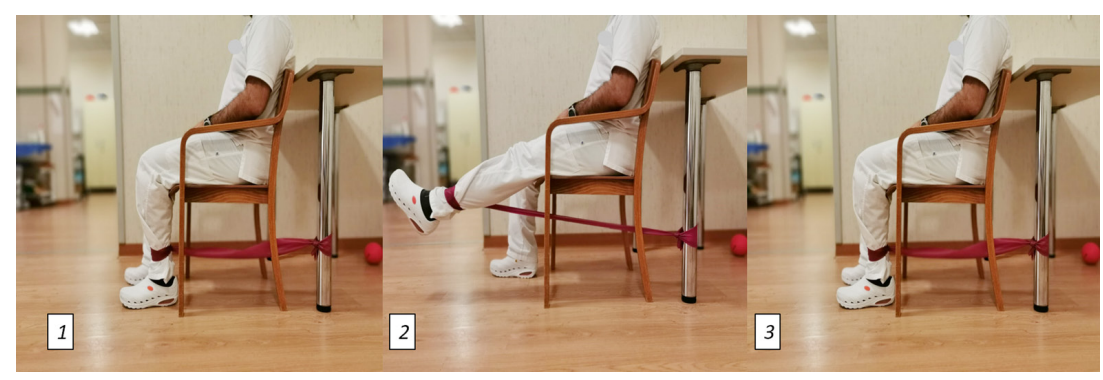

Fig. 2. Home-based exercises for lower limbs.

Exercises to strengthen lower limb muscles (quadriceps, gluteal muscles); 1-3: flexion-extension exercises of the knee (All the exercises illustrated must be performed with shoes with a rubber sole for patient safety).

Table 1. Home-based exercise program

\begin{tabular}{|c|c|c|c|c|}
\hline & 1st week & 2nd week & 3rd week & 4th week \\
\hline $\begin{array}{l}\text { Endurance } \\
\text { training }\end{array}$ & $\begin{array}{l}\text { Walking: warm-up } 2 \text { min, } \\
5 \text { min at } 60 \% / 70 \% \text { of } \mathrm{HR} \\
\max , 3 \text { min cooldown ( } 10 \\
\min ) .\end{array}$ & $\begin{array}{l}\text { IT, } 3 \text { sets of } 3 \text { min: } 1 \mathrm{~min} \\
\text { at } 80 \% \mathrm{HR} \max , 2 \min \\
\text { at } 40 \% / 45 \% \mathrm{HR} \max (10 \\
\text { min). }\end{array}$ & $\begin{array}{l}\text { IT, } 4 \text { sets of } 3 \mathrm{~min}: 1.5 \mathrm{~min} \\
\text { at } 80 \% \mathrm{HR} \max , 1.5 \mathrm{~min} \\
\text { at } 40 \% / 45 \% \mathrm{HR} \max (12 \\
\min ) .\end{array}$ & $\begin{array}{l}\text { IT, } 4 \text { sets of } 3 \text { min: } 2 \min \\
\text { at } 80 \% \mathrm{HR} \max , 1 \mathrm{~min} \\
\text { at } 40 \% / 45 \% \mathrm{HR} \max (12 \\
\text { min). }\end{array}$ \\
\hline $\begin{array}{l}\text { Str } \\
\text { tra }\end{array}$ & $\begin{array}{l}\text { Exercises with elastic } \\
\text { bands: } 2 \text { sets of the number } \\
\text { of repetitions calculated by } \\
\text { the FRT. }\end{array}$ & $\begin{array}{l}\text { Exercises with an elastic } \\
\text { band: } 3 \text { sets of the number } \\
\text { of repetitions calculated } \\
\text { by FRT. }\end{array}$ & $\begin{array}{l}\text { Exercises with an elastic } \\
\text { band: } 4 \text { sets of the number } \\
\text { of repetitions calculated by } \\
\text { the FRT. }\end{array}$ & $\begin{array}{l}\text { Exercises with an elastic } \\
\text { band: } 5 \text { sets of the number } \\
\text { of repetitions calculated by } \\
\text { the FRT. }\end{array}$ \\
\hline $\begin{array}{l}\text { Inspiratory } \\
\text { muscle training }\end{array}$ & $\begin{array}{l}15 \text { inspiratory efforts, with } \\
\text { Threshold set to } 60 \% \text { of } \\
\text { MIP }\end{array}$ & $\begin{array}{l}20 \text { inspiratory efforts, with } \\
\text { Threshold set to } 60 \% \text { of } \\
\text { MIP }\end{array}$ & $\begin{array}{l}25 \text { inspiratory efforts, with } \\
\text { Threshold set to } 60 \% \text { of } \\
\text { MIP }\end{array}$ & $\begin{array}{l}30 \text { inspiratory efforts with } \\
\text { Threshold set to } 60 \% \text { of } \\
\text { MIP }\end{array}$ \\
\hline
\end{tabular}

IT: interval training; HR: heart rate; FRT: fatigue resistance test; MIP: maximal inspiration pressure.

oxygen saturation $\left(\mathrm{SpO}_{2}\right)$ and $\mathrm{HR}$ with finger oximeter. A diary was given to each patient, in which to track adherence to the rehabilitation program, based on the numbers of days and procedures that were performed during the first and second months of rehabilitative treatment. In addition, the patient was able to contact the physiatrist by telephone, if necessary, to help quell any doubts regarding the protocol, which minimized errors and improved adherence.

The sample calculation was obtained for a power of $95 \%$, assuming an alpha error of 0.05 . The calculation was based on the $\mathrm{mBS}$, with a difference of 2 points, assuming a standard deviation (SD) of 1.5 for the $\mathrm{mBS}$ score. The minimal clinically important difference between groups after treatment is approximately 0.9 units ${ }^{43}$. Thus, the analysis yielded 14 patients per group; based on a $20 \%$ potential dropout rate, 20 patients per group were included.

Values are expressed as the median, minimum, and maximum, for continuous variables, and as proportions, for categorical variables, as appropriate. Demographic and clinical data collected at baseline included the following parameters: age and body mass index (BMI), expressed as the median, minimum, and maximum, and gender (female or male). Differences in baseline characteristics between the 2 treatment groups were analyzed by Fisher's exact test or the Mann-Whitney U test, as appropriate. The analysis of evaluation time differences between the 2 groups (TG and CG) was performed through a Friedmann analysis for repeated measures, to determine differences at the different evaluation times between the 2 groups. A subsequent analysis for each parameter, as a pairwise comparison, was performed, with Bonferroni correction. MannWhitney $\mathrm{U}$ test was used for all parameters to evaluate differences between the groups at each time point and differences in the variations $(\Delta)$ between $\mathrm{T} 0$ and $\mathrm{T} 1, \mathrm{~T} 1$ and $\mathrm{T} 2$, and $\mathrm{T} 2$ and $\mathrm{T} 0$. All primary and secondary outcomes were analyzed according to the principle of intention-to-treat. A $p<0.05$ was considered significant. Statistical analyses were performed using SPSS, version 18 (SPSS Inc, Chicago, IL, USA).

\section{RESULTS}

During the treatment and observation period, 6 patients in the TG and 4 patients in the CG did not complete the rehabilitative program due to COPD exacerbation, and 1 patient in the TG was excluded because he did not consistently participate in the HBRP, whereas 2 patients were excluded from the CG after they changed their drug therapy regimens. No adverse events were reported during the rehabilitative treatment or observation periods for the remaining patients in both groups who were included in the study. 
Data from 43 patients were analyzed, including 23 patients in the TG and 20 patients in the CG. A descriptive analysis was performed, as shown in Table 2, for age, BMI, and gender.

Compared with the baseline, the two groups were homogeneous, and matched for age, with a median (range) age of 69 years (58-78), for the TG (14 males and 9 females), and 69 years (63-77), for the CG (11 males, 9 females, $p=0.41)$. No significant differences in BMI were observed between the TG, at $26.53 \mathrm{~kg} / \mathrm{m}^{2}(19-31)$, and the CG, at $27.71 \mathrm{~kg} / \mathrm{m}^{2}(21-31.64$, $\mathrm{p}=0.29$ ). In the TG, 5 patients received $\mathrm{O}_{2}$ therapy, and 1 patient used non-invasive ventilation (NIV), whereas in the CG, 4 patients received $\mathrm{O}_{2}$ therapy, and 2 patients used NIV.

The results showed a significant difference, with $\mathrm{p}<0.05$, with improved values among the TG group, as shown in Table 3 , for the following variables: $\Delta \mathrm{T} 0-\mathrm{T} 1$ and $\Delta \mathrm{T} 0-\mathrm{T} 2$, for diaphragm excursion (Dia-Ex, in cm, Fig. 3), $\Delta \mathrm{T} 0-\mathrm{T} 1$ and $\Delta \mathrm{T} 1-\mathrm{T} 2$ for Dia-Ex MAX (in cm), $\Delta \mathrm{T} 0$-T1 and $\Delta \mathrm{T} 0$-T2 for FVC (in L), $\Delta \mathrm{T} 0$-T1 for 6-MWT (in min), $\Delta \mathrm{T} 0-\mathrm{T} 1, \Delta \mathrm{T} 1-\mathrm{T} 2$, and $\Delta \mathrm{T} 0$-T2 for MIP (in $\mathrm{cmH}_{2} \mathrm{O}$ ), $\Delta \mathrm{T} 1-\mathrm{T} 2$ and $\Delta \mathrm{T} 0$-T2 for MEP (in $\mathrm{cmH}_{2} \mathrm{O}$ ), $\Delta \mathrm{T} 0$-T1 and $\Delta \mathrm{T} 0$-T2 for CAT, and $\Delta \mathrm{T} 0-\mathrm{T} 1$ and $\Delta \mathrm{T} 0$-T2 for mBS.

The within-group analysis demonstrated improvements over time for all parameters in the TG, whereas the results for the CG indicated a constant trend toward increasing respiratory pathology, with improvements observed only for Dia-Ex MAX, FVC, FEV1, for MIP (Table 4) (Fig. 4).

Table 2. Baseline characteristics of the study population

\begin{tabular}{lccc}
\hline & Treatment group & Control group & p-value \\
\hline Age (years) & $69(58-78)$ & $69(63-77)$ & 0.41 \\
Weight $(\mathrm{kg})$ & $74(56-85)$ & $79(60-89)$ & 0.19 \\
Height $(\mathrm{cm})$ & $167(148-186)$ & $166(160-170)$ & 0.54 \\
BMI $\left(\mathrm{kg} / \mathrm{m}^{2}\right)$ & $26.53(19-31)$ & $27.71(21-31.64)$ & 0.29 \\
\hline
\end{tabular}

BMI: body mass index; Values are presented as the median (range).

Table 3. Comparison analysis between groups

\begin{tabular}{|c|c|c|c|c|c|c|c|c|}
\hline \multirow{2}{*}{ Group } & & \multicolumn{4}{|c|}{ Evaluation times } & \multicolumn{3}{|c|}{ POST HOC } \\
\hline & & T0 & $\mathrm{T} 1$ & $\mathrm{~T} 2$ & $\mathrm{p}$-value & p T0-T1 & $\mathrm{p} \mathrm{T} 1-\mathrm{T} 2$ & $\mathrm{p} \mathrm{T0-T2}$ \\
\hline \multirow{9}{*}{ 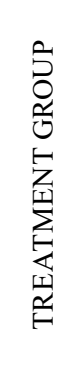 } & Dia-Ex [cm] & $2.2(1.7-4.5)$ & $2.5(1.9-4.9)$ & $2.4(1.8-4.7)$ & $<0.001^{* *}$ & $<0.001^{* *}$ & 1 & $<0.001^{* *}$ \\
\hline & Dia-Ex MAX [cm] & $2.5(1.9-4.9)$ & $2.4(1.8-4.7)$ & $3.5(1.1-7.8)$ & $<0.001^{* *}$ & 1 & $<0.001^{* *}$ & $<0.001 * *$ \\
\hline & $\mathrm{FVC}[\mathrm{L}]$ & $86(58-93)$ & $95(65-135)$ & $93(68-123)$ & $<0.001 * *$ & $<0.001^{* *}$ & 0.55 & $0.001^{*}$ \\
\hline & $\mathrm{FEV}_{1}[\mathrm{~L}]$ & 49 (40-79) & $62(42-85)$ & $60(42-84)$ & $0.018^{*}$ & $0.019^{*}$ & 0.42 & 0.63 \\
\hline & 6-MWT [mt] & $300(50-450)$ & $380(100-500)$ & $360(100-480)$ & $<0.001^{* *}$ & $<0.001^{* *}$ & $0.04 *$ & 0.08 \\
\hline & $\mathrm{MIP}\left[\mathrm{cmH}_{2} \mathrm{O}\right]$ & $69(30-88)$ & $86(39-132)$ & $82(39-130)$ & $<0.001^{* *}$ & $<0.001^{* *}$ & 0.91 & $0.001^{*}$ \\
\hline & $\mathrm{MEP}\left[\mathrm{cmH}_{2} \mathrm{O}\right]$ & $50(24-76)$ & $64(35-89)$ & $62(35-88)$ & $0.017 *$ & $0.024^{*}$ & 0.365 & 0.81 \\
\hline & CAT & $18(10-38)$ & $13(8-34)$ & $12(8-34)$ & $<0.001^{* *}$ & $<0.001 * *$ & 0.63 & $<0.001 * *$ \\
\hline & $\mathrm{mBS}$ & $6(3-8)$ & $5(2-8)$ & $5(2-8)$ & $<0.001^{* *}$ & $0.001 *$ & 1 & $0.005^{*}$ \\
\hline \multirow{9}{*}{ 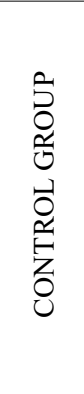 } & Dia-Ex [cm] & $2.25(1.5-2.9)$ & $2.1(1.4-3.1)$ & $2.1(1.4-3.1)$ & 0.06 & - & - & - \\
\hline & Dia-Ex MAX [cm] & $2.1(1.4-3.1)$ & $2.1(1.4-3.1)$ & $2.35(1.4-4.3)$ & $<0.001^{* *}$ & 1 & $<0.001^{* *}$ & $<0.001^{* *}$ \\
\hline & FVC [L] & $79(63-83)$ & $75(68-92)$ & $73(69-86)$ & $<0.001^{* *}$ & $<0.001^{* *}$ & 0.47 & $0.001^{*}$ \\
\hline & $\mathrm{FEV}_{1}[\mathrm{~L}]$ & $53(42-76)$ & $58(44-86)$ & $55(45-76)$ & $0.026^{*}$ & $0.047^{*}$ & 0.2 & 1 \\
\hline & 6-MWT [mt] & $250(170-350)$ & $255(165-400)$ & $257(160-380)$ & 0.18 & - & - & - \\
\hline & MIP $\left[\mathrm{cmH}_{2} \mathrm{O}\right]$ & $70(36-124)$ & $64(23-130)$ & $67.5(30-130)$ & 0.297 & - & - & - \\
\hline & $\mathrm{MEP}\left[\mathrm{cmH}_{2} \mathrm{O}\right]$ & $43.50(17-85)$ & $46.5(16-88)$ & $45.5(16-89)$ & $0.001^{*}$ & $0.001^{*}$ & 0.29 & 0.17 \\
\hline & CAT & $20(14-26)$ & $19(15-26)$ & $19(14-26)$ & 0.192 & - & - & - \\
\hline & $\mathrm{mBS}$ & $5.5(4-7)$ & $5.5(4-7)$ & $5.5(4-7)$ & 0.07 & - & - & - \\
\hline
\end{tabular}

Values are given as the median (min-max).

T0: baseline; T1: after treatment; T2: follow-up; Dia-Ex: diaphragm excursion; FVC: forced vital capacity; FEV1: forced expiratory volume in 1 second; 6-MWT: 6-minute walking test; MIP: maximal inspiration pressure; MEP: maximal expiration pressure; CAT: COPD assessment test; mBS: modified Borg scale; ${ }^{*} \mathrm{p}<0.05 ; * * \mathrm{p}<0.001$. 


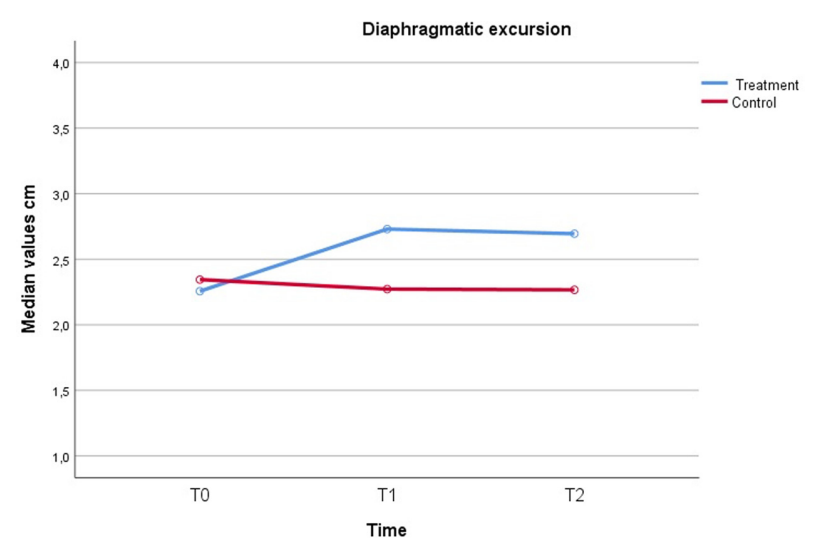

Fig. 3. Flow diagram of the study, according to CONSORT guidelines.

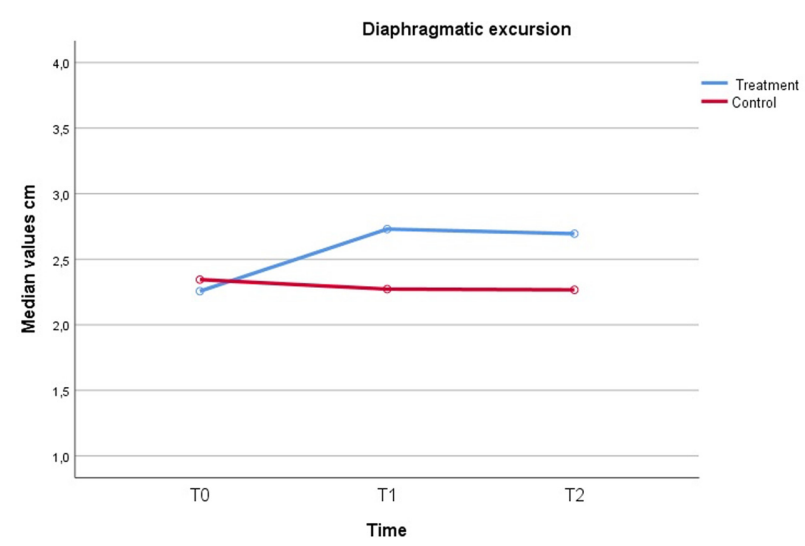

Fig. 4. Interaction plot between groups showing diaphragmatic excursions over time.

Table 4. Time difference $(\Delta)$ between the two groups, with respect to treatment

\begin{tabular}{lccc}
\hline Parameters & Treatment group & Control group & p-value \\
\hline$\Delta$ T0-T1 Dia-Ex [cm] & $0.3(-0.3-1.2)$ & $-0.1(-0.2-0.2)$ & $<0.001^{* *}$ \\
$\Delta$ T1-T2 Dia-Ex [cm] & $0(-0.4-0.2)$ & $0(-0.2-0.1)$ & 0.415 \\
$\Delta$ T0-T2 Dia-Ex [cm] & $0.32(-0.3-1.3)$ & $-0.1(-0.2-0.2)$ & $<0.001^{* *}$ \\
$\Delta$ T0-T1 Dia-Ex MAX [cm] & $0.7(0-2.3)$ & $0.25(-0.5-1.2)$ & $0.029^{*}$ \\
$\Delta$ T1-T2 Dia-Ex MAX [cm] & $-0.01(-1-0.02)$ & $0(-0.1-0.2)$ & $0.026^{*}$ \\
$\Delta$ T0-T2 Dia-Ex MAX [cm] & $0.61(0-2.2)$ & $0.2(-0.5-1.2)$ & 0.094 \\
$\Delta$ T0-T1 FVC [L] & $10(-12-49)$ & $3(-1-24)$ & $0.001^{* *}$ \\
$\Delta$ T1-T2 FVC [L] & $-2(-23-10)$ & $-0.5(-10-1)$ & 0.585 \\
$\Delta$ T0-T2 FVC [L] & $9(-2-37)$ & $2(-7-14)$ & $0.001^{* *}$ \\
$\Delta$ T0-T1 FEV1 [L] & $4(-9-30)$ & $3(-5-44)$ & 0.885 \\
$\Delta$ T1-T2 FEV1 [L] & $-1(-10-3)$ & $-2(-20-1)$ & 0.365 \\
$\Delta$ T0-T2 FEV1 [L] & $3(-7-30)$ & $1(-6-24)$ & 0.693 \\
$\Delta$ T0-T1 6-MWT [mt] & $70(-65-180)$ & $2.5(-60-50)$ & $0.002^{*}$ \\
$\Delta$ T1-T2 6-MWT [mt] & $-20(-50-80)$ & $-5(-100-30)$ & 0.147 \\
$\Delta$ T0-T2 6-MWT [mt] & $50(-85-180)$ & $0(-100-30)$ & $0.002^{*}$ \\
$\Delta$ T0-T1 MIP [cmH $\left.{ }_{2} \mathrm{O}\right]$ & $20(-18-54)$ & $-2(-13-10)$ & $<0.001^{* *}$ \\
$\Delta$ T1-T2 MIP [cmH $\left.{ }_{2} \mathrm{O}\right]$ & $-1(-30-10)$ & $0.5(-5-7)$ & $0.015^{*}$ \\
$\Delta$ T0-T2 MIP [cmH $\left.{ }_{2} \mathrm{O}\right]$ & $16(-11-44)$ & $-1(-6-11)$ & $<0.001^{* *}$ \\
$\Delta$ T0-T1 MEP [cmH $\left.{ }_{2} \mathrm{O}\right]$ & $2(-11-29)$ & $4(-3-8)$ & 0.57 \\
$\Delta$ T1-T2 MEP [cmH $\left.{ }_{2} \mathrm{O}\right]$ & $-1(-20-2)$ & $-1(-10-2)$ & 0.54 \\
$\Delta$ T0-T2 MEP [cmH $\left.\mathrm{cm}_{2} \mathrm{O}\right]$ & $2(-11-28)$ & $2(-7-9)$ & 0.926 \\
$\Delta$ T0-T1 CAT & $4(0-6)$ & $-1(-3-4)$ & $<0.001^{* *}$ \\
$\Delta$ T1-T2 CAT & $0(-5-1)$ & $0(-2-1)$ & 0.094 \\
$\Delta$ T0-T2 CAT & $3(0-7)$ & $0(-3-4)$ & $<0.001^{* *}$ \\
$\Delta$ T0-T1 mBS & $1(-1-3)$ & $0(-1-0)$ & $<0.001^{* *}$ \\
$\Delta$ T1-T2 mBS & $0(-2-1)$ & $0(0-1)$ & 0.065 \\
$\Delta$ T0-T2 mBS & $1(-1-3)$ & $0(-1-0)$ & $<0.001^{* *}$ \\
\hline
\end{tabular}

Values are given as the median (min-max).

$\Delta$ : difference; T0: baseline; T1: after treatment; T2: follow-up; DiaEx: diaphragm excursion; FVC: forced vital capacity; $\mathrm{FEV}_{1}$ : forced expiratory volume in 1 second; 6-MWT: 6-minute walking test; MIP: maximal inspiration pressure; MEP: maximal expiration pressure; CAT: COPD assessment test; mBS: modified Borg scale; ${ }^{*} \mathrm{p}<0.05 ;{ }^{* *} \mathrm{p}<0.001$. 


\section{DISCUSSION}

The principal goal of this study was to investigate the effects of a specific, intensive, and supervised HBRP on dyspnea, based on the mBS score, among COPD patients. The results were encouraging, as comparisons between the TG and CG groups indicated that patients who followed the rehabilitation path demonstrated dyspnea improvements that met the minimal clinically important difference of 0.9 units $^{43}$.

The changes measured between $\mathrm{T} 0$ and $\mathrm{T} 1$ and between $\mathrm{T} 0$ and $\mathrm{T} 2$ showed significant differences in the TG versus the $\mathrm{CG}$, and these results were maintained throughout the follow-up period. In the CG, dyspnea remained constant throughout the 4-month observation period, with a median mBS value of 5.5 (5-7, $\mathrm{p}=0.07)$.

The quality of life perceived by patients, assessed by the CAT scales, showed a significant increase in the TG (median at $\mathrm{T} 0=18$, median at $\mathrm{T} 2=12$ ), with a significant difference between the 2 groups. Several studies indicated that quality of life (QoL) worsened due to the occurrence of dyspnea, anxiety, and depression, which are often present in COPD patients and have been associated with poor physical condition, functional impairment, and comorbidities ${ }^{44)}$.

Therefore, rehabilitative treatment, in this type of chronic and delicate patient, has important validity, not only for strictly clinical and functional aspects but also for social aspects. Other studies in COPD patients have indicated the importance of the respiratory rehabilitation setting from an ecological point of view. Kubincová et al. demonstrated the effectiveness of complex climatotherapy rehabilitation treatments, in a mountainous environment, showing a large treatment effect for the improvement of most quality-of-life dimensions ${ }^{45)}$.

Outpatient rehabilitation is a therapeutic choice for COPD patients, it allows to follow the patient's progress daily and further customize the training program. However, as stated by de Oliveira et al. in most countries, the availability of PR programs is limited, and few patients undergo this treatment modality ${ }^{46)}$. In addition, PR performed in a clinical setting and based on hospital guidelines requires skilled healthcare professionals, equipment and facilities that are expensive to maintain, and this cost contributes to the reduction of the availability of PR at healthcare services ${ }^{46}$.

Respiratory rehabilitation in a home environment allows the patient avoid hospitalization, which also reduced the economic impact. Home-based rehabilitation allows the patient to act as the protagonist of their rehabilitation path, which can be integrated into daily life activities. In addition, the HBPR setting could contribute to changing habits, such as sedentary lifestyles, and help patients learn to appreciate movement by the end of the rehabilitation program.

Based on our study and previous studies, HBPR has favorable impacts on the quality of life in COPD patients. Holland et al. reported the HBPRs utilize minimal resources and require little direct supervision while simultaneously delivering short-term improvements in functional exercise capacity and health-related quality of life, which are at least equivalent to those observed for conventional $\mathrm{PR}^{47}$.

COPD patients often fail to access conventional rehabilitation resources at specialized centers for different reasons (i.e., difficulties with transportation, distance and location of such centers, or lack of perceived benefit $)^{48)}$; therefore, HBRPs may represent a very important resource for COPD patients.

Despite being strongly recommended by treatment guidelines, PR is currently delivered to less than $10 \%$ of COPD patients who would likely benefit from rehabilitation ${ }^{49)}$.

Quality of life and dyspnea improvements are closely related. Patients who do not feel "hungry for air" during activities of daily life can more easily break the vicious cycle in which increased dyspnea leads to a fear of moving and the development of a sedentary lifestyle. In our study, the TG reported good improvements in functional parameters, such as the 6-MWT ( $\Delta$ $\mathrm{T} 0-\mathrm{T} 1, \mathrm{p}=0.002$; and $\Delta \mathrm{T} 0-\mathrm{T} 2, \mathrm{p}=0.002)$ and $\mathrm{FVC}(\Delta \mathrm{T} 0-\mathrm{T} 1, \mathrm{p}=0.001$ and $\Delta \mathrm{T} 0-\mathrm{T} 2, \mathrm{p}=0.001)$, in addition to improvements in dyspnea and quality of life.

Kinesiophobia has been strongly associated with dyspnea perception, fatigue severity, and multisystemic comorbidities in $\mathrm{COPD}^{50)}$. In our rehabilitation program, the physiotherapist and physiatrist, in addition to playing a supervisory role and making themselves available for questions, as necessary, attempted to motivate and encourage the patients, reinforcing the benefits of physical activity. Other groups, such as Meshe et al., have also discussed this important aspect during the respiratory rehabilitation path ${ }^{51)}$.

Further, for the HBPR in this study, simple and safe exercises were recommended. As indicated in the literature ${ }^{15,41,47)}$, the program included an integrated PR program, consisting of an aerobic phase, strength and endurance exercises, and specific exercises for inspiratory muscles. Gosselink et al. suggested that in patients with inspiratory muscle weakness, the addition of IMT to a general exercise training program tended to improve exercise performance ${ }^{15)}$. Basso-Vanelli et al. emphasized that improving inspiratory performance significantly improves dyspnea ${ }^{52}$. The combination of resistance and aerobic training yielded greater increases in muscle endurance and increased resistance training volume compared to concurrent resistance and aerobic training ${ }^{53)}$.

The measurement of the diaphragm range during inspiration may represent a useful method for estimating lung hyperinflation and inspiratory muscle weakness ${ }^{14,16,17)}$.

In COPD patients, thoracic distension occurs due to the volume of air trapped in the lungs, which places the diaphragm in an unfavorable contraction state. The degree of diaphragm shortening during contraction is strongly influenced by the level of diaphragm motor outflow. The improved diaphragmatic excursion on US examination, under basal conditions, was observed 
for the TG, from a median of $2.2 \mathrm{~cm}$ at baseline to 2.5 at $\mathrm{T} 1$ and 2.4 at $\mathrm{T} 2(\mathrm{p}<0.001)$, whereas no change in resting conditions was observed for the $\mathrm{CG}$, with a slight increase observed under conditions of maximum forced respiratory excursions. US examination of the diaphragm muscle represents a non-invasive method that could be used to monitor the response to rehabilitation treatments. Further, our results showed simultaneous improvements in the range of diaphragmatic excursions on US examination and the MIP in the TG.

In the TG, increases in MIP and MEP were measured, whereas in the CG, a small increase in MEP but not MIP was observed. The differences between the 2 groups were significant immediately after treatment for MIP but not for MEP. These results suggest 2 hypotheses. The first hypothesis is that CG patients continued to activate their respiratory muscles in compensatory manners, resorting increasingly on the support of expiratory muscles. The second hypothesis is that to maintain the good activation of inspiratory muscles (diaphragm), the exercises must be performed constantly and continuously. At T2, the MIP value of the TG was not maintained at the level observed at T1. Kim et al. reported as the strength of the inspiratory muscles may better reflect the severity of COPD disease when compared to that of expiratory muscles ${ }^{54)}$.

Our results suggested that an intensive and supervised HBPR that is performed consistently and includes elements of specific exercises designed to improve inspiratory endurance, aerobic, and strength training was effective for improving diaphragmatic function during the respiratory cycle, as assessed by US evaluation. The weekly supervision provided by the physiotherapist ensured good adherence to treatment among the patients, and no adverse effects related to the execution of the proposed program were reported during the rehabilitative treatment. The TG also demonstrated improved clinical and functional parameters compared with the $\mathrm{CG}$, including the reduction of dyspnea and improved quality of life, demonstrating the beneficial effects of HBPR for mild COPD patients when implemented as a tailored, patient self-management, supervised program.

Suitable HBPR programs must be developed, and future studies should evaluate the long-term effects of these programs with respect to pulmonary function.

In terms of study limitations and prospects, our study presented a period of 4 months, with a short follow-up of only 2 months. Although this short time frame could represent a limit, it was chosen to reduce the risks of exacerbations, which would require the exclusion of additional patients from the study. However, even with this short time frame, 12 patients were excluded during our study due to the exacerbation of respiratory failure. The small size of the sample did not permit additional analyses to be performed, such as correlation analyses.

\section{Data availability statement}

The clinical data used to support the findings of this study are available from the corresponding author upon request.

\section{Authors' contributions}

Study conceptualization and design: TP and AS; Literature search: LP and FA; Writing first draft: TP; Revision of the first draft: RS; Supervision: RS and RGB; statistical analysis: MM and AB; physiotherapy: ADM and NG; evaluation scale: GPP and NG. All authors read and approved the final version of the manuscript.

\section{Funding}

The authors report no involvement by sponsor that could have influenced the outcome of this research.

\section{Conflict of interest}

All the authors declare no conflicts of interest regarding this paper.

\section{ACKNOWLEDGEMENT}

We thank Lisa Giles, PhD, from Blue Pencil Science (http://www.bluepencilscience.com/) for editing an English draft of this manuscript.

\section{REFERENCES}

1) Rossi A, Butorac-Petanjek B, Chilosi M, et al.: Chronic obstructive pulmonary disease with mild airflow limitation: current knowledge and proposal for future research-a consensus document from six scientific societies. Int J Chron Obstruct Pulmon Dis, 2017, 12: 2593-2610. [Medline] [CrossRef]

2) Vogelmeier CF, Criner GJ, Martínez FJ, et al.: Global strategy for the diagnosis, management, and prevention of chronic obstructive lung disease 2017 report: GOLD executive summary. Am J Respir Crit Care Med, 2017, 195: 557-582. [Medline] [CrossRef]

3) Adeloye D, Chua S, Lee C, et al. Global Health Epidemiology Reference Group (GHERG): Global and regional estimates of COPD prevalence: systematic review and meta-analysis. J Glob Health, 2015, 5: 020415. [Medline] [CrossRef]

4) GBD 2015 Chronic Respiratory Disease Collaborators: Global, regional, and national deaths, prevalence, disability-adjusted life years, and years lived with disability for chronic obstructive pulmonary disease and asthma, 1990-2015: a systematic analysis for the Global Burden of Disease Study 2015. Lancet Respir 
Med, 2017, 5: 691-706. [Medline] [CrossRef]

5) Gagnon P, Guenette JA, Langer D, et al.: Pathogenesis of hyperinflation in chronic obstructive pulmonary disease. Int J Chron Obstruct Pulmon Dis, 2014, 9: 187-201. [Medline]

6) Bernard S, LeBlanc P, Whittom F, et al.: Peripheral muscle weakness in patients with chronic obstructive pulmonary disease. Am J Respir Crit Care Med, 1998, 158: 629-634. [Medline] [CrossRef]

7) Landry MD, Hamdan E, Al Mazeedi S, et al.: The precarious balance between 'supply' and 'demand' for health care: the increasing global demand for rehabilitation service for individuals living with chronic obstructive pulmonary disease. Int J Chron Obstruct Pulmon Dis, 2008, 3: 393-396. [Medline] [CrossRef]

8) Zeng Y, Jiang F, Chen Y, et al.: Exercise assessments and trainings of pulmonary rehabilitation in COPD: a literature review. Int J Chron Obstruct Pulmon Dis, 2018, 13: 2013-2023. [Medline] [CrossRef]

9) Marotta N, Demeco A, Moggio L, et al.: Comparative effectiveness of breathing exercises in patients with chronic obstructive pulmonary disease. Complement Ther Clin Pract, 2020, 41: 101260. [Medline] [CrossRef]

10) Dias FD, Sampaio LM, da Silva GA, et al.: Home-based pulmonary rehabilitation in patients with chronic obstructive pulmonary disease: a randomized clinical trial. Int J Chron Obstruct Pulmon Dis, 2013, 8: 537-544. [Medline] [CrossRef]

11) Liu XL, Tan JY, Wang T, et al.: Effectiveness of home-based pulmonary rehabilitation for patients with chronic obstructive pulmonary disease: a meta-analysis of randomized controlled trials. Rehabil Nurs, 2014, 39: 36-59. [Medline] [CrossRef]

12) Maltais F, Bourbeau J, Shapiro S, et al. Chronic Obstructive Pulmonary Disease Axis of Respiratory Health Network, Fonds de recherche en santé du Québec: Effects of home-based pulmonary rehabilitation in patients with chronic obstructive pulmonary disease: a randomized trial. Ann Intern Med, 2008, 149: 869-878. [Medline] [CrossRef]

13) do Nascimento ES, Sampaio LM, Peixoto-Souza FS, et al.: Home-based pulmonary rehabilitation improves clinical features and systemic inflammation in chronic obstructive pulmonary disease patients. Int J Chron Obstruct Pulmon Dis, 2015, 10: 645-653. [Medline]

14) Smargiassi A, Inchingolo R, Tagliaboschi L, et al.: Ultrasonographic assessment of the diaphragm in chronic obstructive pulmonary disease patients: relationships with pulmonary function and the influence of body composition—a pilot study. Respiration, 2014, 87: 364-371. [Medline] [CrossRef]

15) Gosselink R, De Vos J, van den Heuvel SP, et al.: Impact of inspiratory muscle training in patients with COPD: what is the evidence? Eur Respir J, 2011, 37: 416-425. [Medline] [CrossRef]

16) Crimi C, Heffler E, Augelletti T, et al.: Utility of ultrasound assessment of diaphragmatic function before and after pulmonary rehabilitation in COPD patients. Int J Chron Obstruct Pulmon Dis, 2018, 13: 3131-3139. [Medline] [CrossRef]

17) Vestbo J, Hurd SS, Agustí AG, et al.: Global strategy for the diagnosis, management, and prevention of chronic obstructive pulmonary disease: GOLD executive summary. Am J Respir Crit Care Med, 2013, 187: 347-365. [Medline] [CrossRef]

18) Sarwal A, Walker FO, Cartwright MS: Neuromuscular ultrasound for evaluation of the diaphragm. Muscle Nerve, 2013, 47: 319-329. [Medline] [CrossRef]

19) Supinski GS, Morris PE, Dhar S, et al.: Diaphragm dysfunction in critical illness. Chest, 2018, 153: 1040-1051. [Medline] [CrossRef]

20) Gerscovich EO, Cronan M, McGahan JP, et al.: Ultrasonographic evaluation of diaphragmatic motion. J Ultrasound Med, 2001, 20: 597-604. [Medline] [CrossRef]

21) Matamis D, Soilemezi E, Tsagourias M, et al.: Sonographic evaluation of the diaphragm in critically ill patients. Technique and clinical applications. Intensive Care Med, 2013, 39: 801-810. [Medline] [CrossRef]

22) O'Donnell DE, Voduc N, Fitzpatrick M, et al.: Effect of salmeterol on the ventilatory response to exercise in chronic obstructive pulmonary disease. Eur Respir J, 2004, 24: 86-94. [Medline] [CrossRef]

23) O’Donnell DE: Hyperinflation, dyspnea, and exercise intolerance in chronic obstructive pulmonary disease. Proc Am Thorac Soc, 2006, 3: 180-184. [Medline] [CrossRef]

24) von Elm E, Altman DG, Egger M, et al. STROBE Initiative: The Strengthening the Reporting of Observational Studies in Epidemiology (STROBE) statement: guidelines for reporting observational studies. Ann Intern Med, 2007, 147: 573-577. [Medline] [CrossRef]

25) Global Initiative for Chronic Obstructive Lung Disease: Global strategy for the diagnosis, management, and prevention of chronic obstructive pulmonary disease - 2020 report. www.goldcopd.org Last update December 2019.

26) Antenora F, Fantini R, Iattoni A, et al.: Prevalence and outcomes of diaphragmatic dysfunction assessed by ultrasound technology during acute exacerbation of COPD: a pilot study. Respirology, 2017, 22: 338-344. [Medline] [CrossRef]

27) Zambon M, Greco M, Bocchino S, et al.: Assessment of diaphragmatic dysfunction in the critically ill patient with ultrasound: a systematic review. Intensive Care Med, 2017, 43: 29-38. [Medline] [CrossRef]

28) Roriz D, Abreu I, Belo-Soares P, et al.: Ultrasound in the evaluation of diaphragm. ECR, 2015.

29) Boussuges A, Gole Y, Blanc P: Diaphragmatic motion studied by m-mode ultrasonography: methods, reproducibility, and normal values. Chest, 2009, 135: 391-400. [Medline] [CrossRef]

30) Trzaska-Sobczak M, Brożek G, Farnik M, et al.: Evaluation of COPD progression based on spirometry and exercise capacity. Pneumonol Alergol Pol, 2013, 81: 288-293. [Medline]

31) Morris JF: Spirometry in the evaluation of pulmonary function. West J Med, 1976, 125: 110-118. [Medline]

32) Milic-Emili J: Measurements of pressures in respiratory physiology. Measurements of pressures in respiratory physiology. In: Techniques in life sciences, vol. P4/JI. Amsterdam: Elsevier, 1984, pp 1-22.

33) Damato S, Bonatti C, Frigo V, et al.: Validation of the Clinical COPD questionnaire in Italian language. Health Qual Life Outcomes, 2005, 3: 9. [Medline] [CrossRef]

34) Kendrick KR, Baxi SC, Smith RM: Usefulness of the modified 0-10 Borg scale in assessing the degree of dyspnea in patients with COPD and asthma. J Emerg Nurs, 2000, 26: 216-222. [Medline] [CrossRef]

35) Troosters T, Vilaro J, Rabinovich R, et al.: Physiological responses to the 6-min walk test in patients with chronic obstructive pulmonary disease. Eur Respir J, 2002, 20: 564-569. [Medline] [CrossRef]

36) Cote CG, Casanova C, Marín JM, et al.: Validation and comparison of reference equations for the 6-min walk distance test. Eur Respir J, 2008, 31: 571-578. [Medline] [CrossRef] 
37) Pitta F, Troosters T, Spruit MA, et al.: Characteristics of physical activities in daily life in chronic obstructive pulmonary disease. Am J Respir Crit Care Med, 2005, 171: 972-977. [Medline] [CrossRef]

38) Dal Negro RW, Bonadiman L, Turco P: Sensitivity of the COPD assessment test (CAT questionnaire) investigated in a population of 681 consecutive patients referring to a lung clinic: the first Italian specific study. Multidiscip Respir Med, 2014, 9: 15. [Medline] [CrossRef]

39) Ramos EM, de Toledo-Arruda AC, Fosco LC, et al.: The effects of elastic tubing-based resistance training compared with conventional resistance training in patients with moderate chronic obstructive pulmonary disease: a randomized clinical trial. Clin Rehabil, 2014, 28: 1096-1106. [Medline] [CrossRef]

40) Ambrosino N, Bertella E: Lifestyle interventions in prevention and comprehensive management of COPD. Breathe (Sheff), 2018, 14: 186-194. [Medline] [CrossRef]

41) Gloeckl R, Marinov B, Pitta F: Practical recommendations for exercise training in patients with COPD. Eur Respir Rev, 2013, 22: 178-186. [Medline] [CrossRef]

42) Gosselink R, Wagenaar RC, Decramer M: Reliability of a commercially available threshold loading device in healthy subjects and in patients with chronic obstructive pulmonary disease. Thorax, 1996, 51: 601-605. [Medline] [CrossRef]

43) Khair RM, Nwaneri C, Damico RL, et al.: The minimal important difference in Borg dyspnea score in pulmonary arterial hypertension. Ann Am Thorac Soc, 2016, 13: 842-849. [Medline] [CrossRef]

44) Maurer J, Rebbapragada V, Borson S, et al. ACCP Workshop Panel on Anxiety and Depression in COPD: Anxiety and depression in COPD: current understanding, unanswered questions, and research needs. Chest, 2008, 134: 43S-56S. [Medline] [CrossRef]

45) Kubincová A, Takáč P, Kendrová L, et al.: The effect of pulmonary rehabilitation in mountain environment on exercise capacity and quality of life in patients with Chronic Obstructive Pulmonary Disease (COPD) and chronic bronchitis. Med Sci Monit, 2018, 24: 6375-6386. [Medline] [CrossRef]

46) Mendes de Oliveira JC, Studart Leitão Filho FS, Malosa Sampaio LM, et al.: Outpatient vs. home-based pulmonary rehabilitation in COPD: a randomized controlled trial. Multidiscip Respir Med, 2010, 5: 401-408. [Medline] [CrossRef]

47) Holland AE, Mahal A, Hill CJ, et al.: Home-based rehabilitation for COPD using minimal resources: a randomised, controlled equivalence trial. Thorax, 2017, 72: 57-65. [Medline] [CrossRef]

48) Keating A, Lee A, Holland AE: What prevents people with chronic obstructive pulmonary disease from attending pulmonary rehabilitation? A systematic review. Chron Respir Dis, 2011, 8: 89-99. [Medline] [CrossRef]

49) Weldam SW, Lammers JW, Heijmans MJ, et al.: Perceived quality of life in chronic obstructive pulmonary disease patients: a cross-sectional study in primary care on the role of illness perceptions. BMC Fam Pract, 2014, 15: 140. [Medline] [CrossRef]

50) Vardar-Yagli N, Calik-Kutukcu E, Saglam M, et al.: The relationship between fear of movement, pain and fatigue severity, dyspnea level and comorbidities in patients with chronic obstructive pulmonary disease. Disabil Rehabil, 2019, 41: 2159-2163. [Medline] [CrossRef]

51) Meshe OF, Claydon LS, Bungay H, et al.: The relationship between physical activity and health status in patients with chronic obstructive pulmonary disease following pulmonary rehabilitation. Disabil Rehabil, 2017, 39: 746-756. [Medline] [CrossRef]

52) Basso-Vanelli RP, Di Lorenzo VA, Labadessa IG, et al.: Effects of inspiratory muscle training and calisthenics-and-breathing exercises in COPD with and without respiratory muscle weakness. Respir Care, 2016, 61: 50-60. [Medline] [CrossRef]

53) Covey MK, Collins EG, Reynertson SI, et al.: Resistance training as a preconditioning strategy for enhancing aerobic exercise training outcomes in COPD. Respir Med, 2014, 108: 1141-1152. [Medline] [CrossRef]

54) Kim NS, Seo JH, Ko MH, et al.: Respiratory muscle strength in patients with Chronic Obstructive Pulmonary Disease. Ann Rehabil Med, 2017 , 41 : 659-666. [Medline] [CrossRef] 